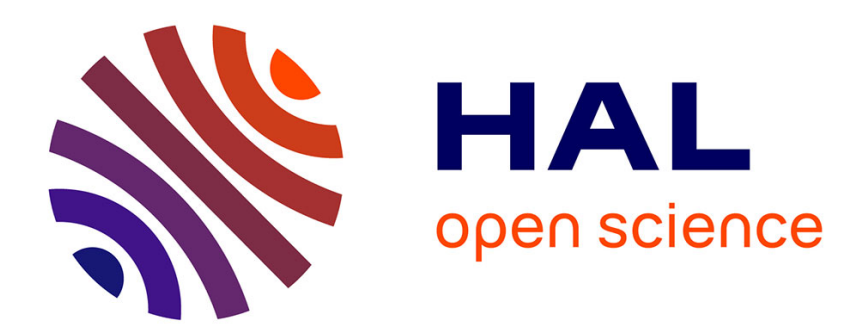

\title{
Le futuroscope et les politiques touristiques du département de la Vienne
}

\author{
Olivier Dehoorne
}

\section{To cite this version:}

Olivier Dehoorne. Le futuroscope et les politiques touristiques du département de la Vienne. Norois, 1998, 45 (179), pp.531-542. hal-01406635

\section{HAL Id: hal-01406635 \\ https://hal.science/hal-01406635}

Submitted on 8 Dec 2016

HAL is a multi-disciplinary open access archive for the deposit and dissemination of scientific research documents, whether they are published or not. The documents may come from teaching and research institutions in France or abroad, or from public or private research centers.
L'archive ouverte pluridisciplinaire HAL, est destinée au dépôt et à la diffusion de documents scientifiques de niveau recherche, publiés ou non, émanant des établissements d'enseignement et de recherche français ou étrangers, des laboratoires publics ou privés. 


\title{
Le Futuroscope et les politiques touristiques du département de la
}

\section{Vienne}

\section{Olivier Dehoorne}

\begin{abstract}
As a French rural department with a small proportion of visitors, the department of the Vienne launched an ambitions development of tourism on the early 90's. The exploitation of this new economic sector is based on the creation of the theme park of the Futuroscope which is situated a few kilometres away from the town of Poitiers. It is from this centre, which now receives over three million visitors each year, that the Department intends to develop half of its territory with its countryside then looked on as an hinterland against this centre of tourism attraction.
\end{abstract}

\section{Résumé}

Département rural faiblement fréquenté, la Vienne a entrepris un développement touristique ambitieux au début des années 90. La mise en valeur de ce nouveau secteur économique repose sur la création du parc d' attraction du Futuroscope implanté à quelques kilomètres de Poitiers. $C$ est à partir de ce rôle qui reçoit désormais plus de trois millions de visiteurs par an que le Département entend aménager la totalité de son territoire, les campagnes de la Vienne faisant alors figure d' arrière-pays vis-àvis de cette nouvelle centr alité touristique.

\section{Citer ce document / Cite this document :}

Dehoorne Olivier. Le Futuroscope et les politiques touristiques du département de la Vienne. In: Norois, n¹79, JuilletSeptembre 1998. pp. 531-542;

doi : $10.3406 /$ noroi.1998.6894

http://www.persee.fr/doc/noroi_0029-182x_1998_num_179_1_6894

Document généré le 12/06/2016 
Norois, 1998 , Poitiers, $t .45, \mathrm{n}^{\circ} 179$, p. $531-542$

\title{
LE FUTUROSCOPE ET LES POLITIQUES TOURISTIQUES DU DÉPARTEMENT DE LA VIENNE
}

\author{
par Olivier DEHOORNE \\ Département de Géographie \\ Université de Poitiers \\ 97, Avenue du Recteur Pineau \\ 86022 Poitiers Cedex
}

\begin{abstract}
RÉSUMÉ
Département rural faiblement fiéquenté, la Vienne a entrepris un développement touristique ambitieux au début des années 90. La mise en valeur de ce nouveau secteur économique repose sur la création du parc d'attraction du Futuroscope implanté à quelques kilomètres de Poitiers. C'est à partir de ce rôle qui reçoit désormais plus de trois millions de visiteurs par an que le Département entend aménager la totalité de son territoire, les campagnes de la Vienne faisant alors figure d'arrière-pays vis-à-vis de cette nouvelle centralité touristique.
\end{abstract}

\section{ABSTRACT}

As a French rural department with a small proportion of visitors, the department of the Vienne launched an ambitions development of tourism on the early 90's. The exploitation of this new economic sector is based on the creation of the theme park of the Futuroscope which is situated a few kilometres away from the town of Poitiers. It is from this centre, which now receives over three million visitors each year, that the Department intends to develop half of its territory with its countryside then looked on as an hinterland against this centre of tourism attraction.

L'essor touristique du département de la Vienne ne date que d'une dizaine d'années et relève d'une stratégie originale. Jusqu'à la fin de la décennie 1980, les campagnes de la Vienne ne connaissaient qu'une fréquentation faible et diffuse. En effet ce département ne se distingue par aucun grand site naturel. Certes, il convient d'évoquer l'intérêt de certains paysages ruraux, notamment dans la partie sud-orientale du département traversée par les vallées de la Gartempe et de la Vienne où les vallonnements annoncent les hauteurs limousines.

Mots-clés : Futuroscope. Département de la Vienne. Parc d'attraction. Tourisme rural. Aménagement rural.

Key words : Futuroscope. Department of the Vienne. Theme park. Rural tourism. Rural development. 
En ce qui concerne le patrimoine historique, cette partie du Haut-Poitou recense de nombreux témoignages de l'art roman, parmi lesquels quelques édifices, plus ou moins bien valorisés, comme les abbayes de Charroux et de Saint-Savin ou la cathédrale Notre-Dame de Poitiers. Il convient de retenir également l'activité de la modeste station thermale de la Roche-Posay qui accueille près de 10000 curistes chaque année.

En fait, ce département rural, qui est avant tout une zone de passage, n'avait rien de transcendant sur le plan touristique. Et sur le plan régional, la Vienne était l'espace le moins bien loti : loin du littoral de la Charente-Maritime, le département n'avait pas d'attrait majeur comme les Deux-Sèvres avec le Marais Poitevin ou de la Charente avec son fleuve et le cognac.

C'est dans ce contexte qu'en 1983 le Conseil général de la Vienne décida la création d'un grand pôle économique et touristique : le parc du Futuroscope dont l'activité commença dès 1987. Le Département entendait ainsi insuffler un nouveau dynamisme à une économie somnolente en mettant à profit les nouvelles responsabilités que lui octroyaient les lois de la décentralisation.

Après dix années de fonctionnement du parc, force est de constater que l'activité touristique a considérablement évolué dans la Vienne, affirmant son originalité avec la constitution d'une centralité bâtie autour du Futuroscope. Et le Département entend profiter du succès de ce parc d'attraction pour entreprendre un aménagement touristique de l'ensemble de son territoire.

\section{I. - LE PARC DU FUTUROSCOPE : LE FONDEMENT D'UNE CEN- TRALITÉ TERTIAIRE ET TOURISTIQUE}

Situé à une dizaine de kilomètres de Poitiers, en bordure nord de l'agglomération, le Futuroscope, ou parc européen de l'image, s'étend sur 250 hectares entre la route nationale 10 et l'autoroute 10 . Cet espace ludique est consacré à la découverte des nouvelles techniques dans les domaines de la communication et de l'image.

Contrastant avec les paysages de plaines du Haut-Poitou, cet ensemble affichc son originalité par d'imposant volumes architecturaux aux allures futuristes qui ne manquent pas d'étonner le visiteur ou le simple automobiliste qui emprunte "l'Aquitaine". Symbole du parc, le pavillon du Futuroscope se présente sous la forme d'un prisme de verre géant, surmonté d'une sphère blanche de dix-sept mètres de diamètres. Quant au pavillon du cinéma - dit kinémax -, son volume ressemble à des cristaux de roche géants, recouverts de miroirs, dressés en oblique. C'est dans cet espace que sont projetés des films sur le plus grand écran plat d'Europe $\left(600 \mathrm{~m}^{2}\right)$ depuis l'ouverture du parc (1).

L'édification du parc se fait progressivement. En 1988, ce fut l'ouverture d'un cinéma dynamique ( 45 places) où les mouvements des sièges sont synchronisés avec l'action du film. Ce succès acquis, un second de 80 places ouvrit six ans plus tard. Un cinéma en relief fonctionne depuis 1989, d'autres projections sont faites sur un écran semi-sphérique de $180^{\circ}$ dès l'année suivante, ou encore sur un écran perpendiculaire - dit le tapis magique en raison du second écran placé sous les sièges - à partir de 1992. Les innovations se poursuivent avec un écran hémisphé-

(1) Cf Pitie (J.), 1988. - «Le parc du Futuroscope : un espace original en PoitouCharentes " in Norois, $\mathrm{n}^{\circ} 139$, p. 367-372 et Bareau (G), 1996. - Le pôle du Futuroscope : aménagement de l'espace et implications économiques, mémoire de maîtrise (dir. J. Soumagne), $266 \mathrm{p}$. 
rique de $800 \mathrm{~m}^{2}$ en 1993, puis un écran semi-cylindrique un an plus tard. Il y a donc un renouvellement constant des technologies, les nouveautés qui apparaissent dans les parcs d'attraction américain et japonais sont reproduites au Futuroscope. parmi les dernières réalisations, on peut citer l'ouverture en 1997 d'un "Cyberavenue » de $800 \mathrm{~m}^{2}$, entre l'internet et les jeux interactifs.

Naturellement, le parc est agrémenté d'espaces ludiques réservés aux plus jeunes et d'un petit plan d'eau dominé par des gradins de 600 places qui devient la scène d'un théâtre alphanumérique pendant les soirées d'été. Ces spectacles permettent de retenir davantage une clientèle susceptible de dîner dans le parc. D'ailleurs le Futuroscope compte en 1997 une vingtaine de restaurants et de points de vente alimentaire (contre 3 en 1987). Et l'appareil commercial cst complété par un débil de tabac et une vingtaine de boutiques.

La progression de la fréquentation du parc d'attraction est spectaculaire : 222500 visiteurs pour la première saison, près d'un demi-million l'année suivante et un million en 1991. Enfin 2,8 millions de visiteurs furent accueillis en 1995 et en 1996. La vente d'un billet valable deux jours connaît un succès croissant : $25 \%$ ont opté pour cette formule en $1992,50 \%$ en 1995 et $56 \%$ en 1996.

TABLEAU I. - Les dix premières années d'activité du Futuroscope. (sources: Futuroscope)

\begin{tabular}{|l|c|c|c|c|c|c|c|c|c|c|}
\hline & 1987 & 1988 & 1989 & 1990 & 1991 & 1992 & 1993 & 1994 & 1995 & 1996 \\
\hline $\begin{array}{l}\text { Nombre de visiteurs } \\
\text { (en milliers) }\end{array}$ & 225 & 450 & 750 & 900 & 1000 & 1300 & 1950 & 2500 & 2800 & 2800 \\
\hline $\begin{array}{l}\text { Chiffres d'affaires } \\
\text { (en millions de francs) }\end{array}$ & 7,5 & 26 & 69 & 90 & 125 & 175 & 300 & 430 & 500 & 570 \\
\hline
\end{tabular}

Les résultats financiers enregistrent la même évolution. Le chiffres d'affaires qui était de 7,5 millions de francs en 1987 a rapidement progressé, atteignant 125 millions en 1991 puis 500 en 1995 et 570 en 1996 pour un bénéfice net de 23 millions de francs. Au cours de cette decennie, les tarifs ont subi une augmentation qui, en tenant compte des différentes formules proposées selon les saisons, est comprise entre $108 \%$ et $146 \%$.

Au sein du parc d'attraction, ouvert toute l'année, depuis 1995, travaillent près de 1200 personnes auxquelles il convient d'associer celles installées dans les directions parisiennes de la commission et du marketing. Au total, 350 salariés bénéficient d'un contrat à durée indéterminée, les autres sont des vacataires ou ne font que des extra prisés par les étudiants poitevins.

Enfin il est à noter que la maîtrise d'œuvre du parc fut assurée par le Conseil Général de la Vienne. Une société d'économie mixte au capital de six millions de francs fut constituée ; elles est sous contrôle du Département à $70 \%$, le reste réunit entre autre la Compagnie Générale des Eaux, la Compagnie financière de Suez et le groupe Accor.

En l'espace de dix années, le parc d'attraction du Futuroscope est devenu un évènement permanent qui contribue à forger une nouvelle image du département. $\mathrm{Et}$, au-delà de l'aspect récréatif, il faut préciser que le site du Futuroscope comporte aussi une zone d'activité technologique ainsi qu'une aire de formation et de 
recherche. La réalisation d'un téléport, opérationnel dès la fin 198, fut déterminante pour attirer des entreprises de pointe dans les secteurs de l'image et de la télécommunications. Un complexe industriel de 1200 hectares est en cours d'aménagement. Pour l'heure près de soixante-dix entreprises sont implantées dans la zone du Futuroscope ; et l'une des dernières en date est le centre d'exploitation de la Messagerie Tam-Tam qui vient de transférer son entreprise parisienne de Montrouge et de recruter localement une centaine de personnes.

Enfin, sur le plan départemental, l'aménagement de cet ensemble contribue à la consolidation d'une concentration déjà existante : l'axe urbain qui se dessine entre Poitiers et Châtellerault. Au cours des dernières décennies, c'est le secteur qui a accueilli l'essentiel des nouvelles activités secondaires et tertiaires du département. Ce couloir édifié le long de l'autoroute et de la principale route nationale du département regroupe 175000 habitants, soit $45 \%$ de la population de la Vienne sur un dixième de son espace.

Outre la consolidation de cet espace économique, la nouveauté résultant du Futu roscope est bien entendu le développement d'une fonction touristique de grande envergure, avec le parc d'attraction, instrument de promotion de la Vienne, qui doit impulser une nouvelle dynamique dans ce département rural.

\section{II. - RENOUVELLEMENT DE L'OFFRE ET REDISTRIBUTION SPA- TIALE}

Durant les décennies précécentes, l'offre touristique de la Vienne ne se démarquait guère de celles des départements ruraux limitrophes; elle connaissait même une érosion graduelle : seulement 19650 lits marchands en 1991 contre plus de 21000 en 1988.

Cependant, à partir de 1990, la disparition des structures d'accueil les plus anciennes, souvent inadaptées à la demande fut compensée par la création de nouveaux hébergements de qualité meilleure. Et, naturellement cette évolution se traduit par une nouvelle géographie de l'offre dans le département où se dessine une rupture entre la centralité et le reste des campagnes.

Depuis 1991, le parc d'accueil a progressé de $23 \%$, soit un gain de 4500 lits. Désormais la Vienne détient $10,6 \%$ de l'offre régionale (contre $7 \%$ en 1991); une offre qui reste dominée par la Charente-Maritime avec 176000 lits soit $78 \%$ du total régional).

Le secteur de l'hôtellerie a connu les transformations les plus significatives : sur les 151 établissements recensés en 1987, seulement 66 sont toujours en activité. En 1993, le département ne comptait plus que 94 hôtels, mais trois ans plus tard il y en a 125. Au cours de cette période, plus de 900 chambres nouvelles sont commercialisées chaque année (954 en 1994 puis 915 en 1995). Toutefois les résultats de 1996, avec la création de 411 chambres, marquent une rupture dans cette croissance et un projet hôtelier de 1000 chambres sur le site du Futuroscope vient d'être abandonné.

Le renouvellement de ce secteur, qui semble réalisé pour l'essentiel, s'est traduit par une légère progression de la qualité de l'offre qui reste moyenne avec $80 \%$ des lits classés une et deux étoiles.

Suivant une tendance générale, la petite hôtellerie et les hôtels de préfecture, qui étaient avant tout les lieux de rencontres dans les campagnes, associant souvent les fonctions de café et de restaurant, voire de bureau de tabac, disparaissent régulièrement. Ils ne totalisent plus que 1790 chambres en 1995 contre 2112 en 1991 . 


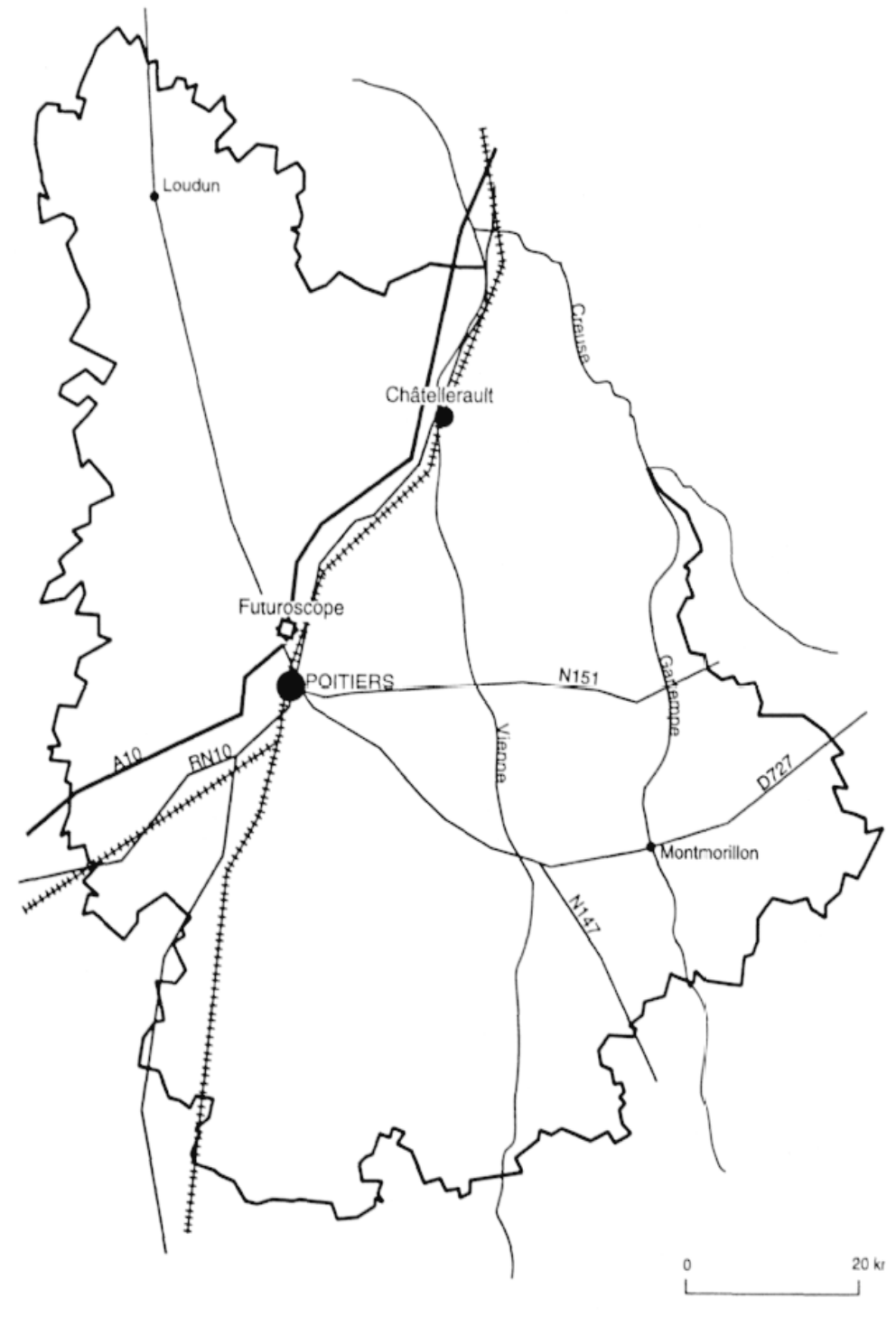

FIG. 1. - Le département de la Vienne.

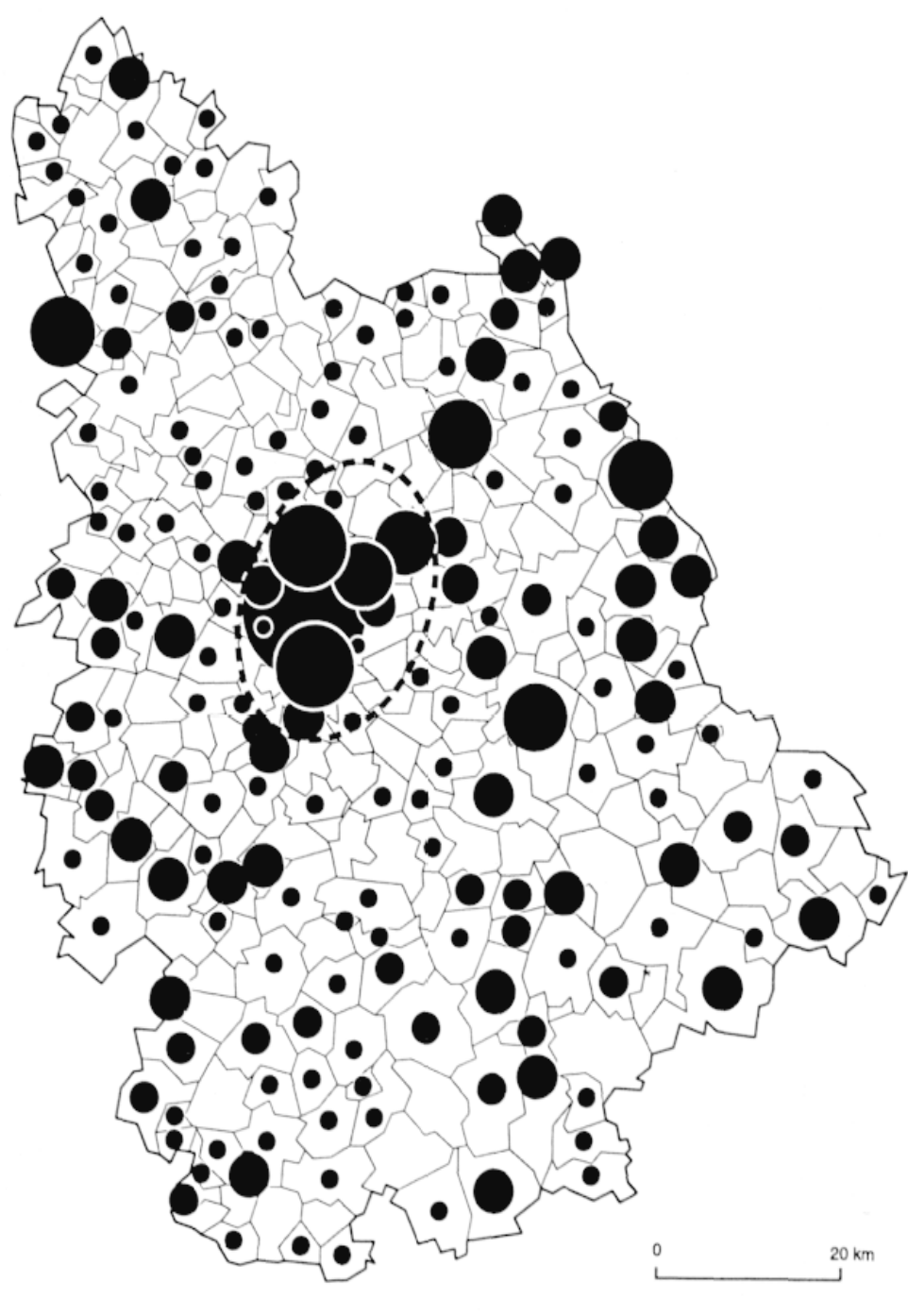

Nombre des lits représentés

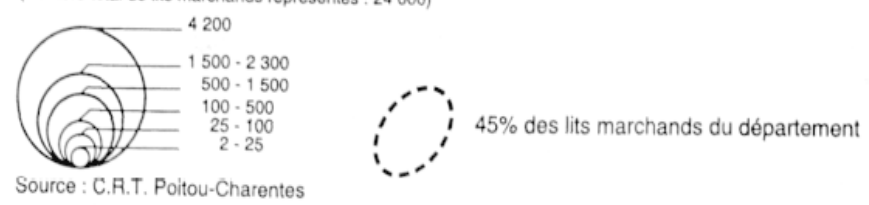

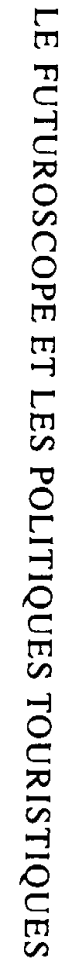

$\underset{w}{u}$ 
Et, plus généralement, les petits hôtels, éloignés des grands axes, appartenant à des campagnes peu fréquentées, ferment régulièrement. Près d'une soixantaine d'établissements ont ainsi cessé leur activité au cours des sept dernières années.

Fort logiquement, le renouvellement de l'offre s'accompagne d'une redictribution au profit de la centralité : cette nouvelle offre hôtelière se concentre aux abords du parc d'attraction et sur le site même du Futuroscope qui compte 1750 chambres en 1997.

La concentration est moins significative dans le secteur de l'hôtellerie de plein air, notamment en raison des campings implantés de longue date dans les vallées de la vallées de la Vienne et de la Gartempe ainsi qu'aux abords de la R.N. 10. Cependant, si l'on ne considère que les quinze campings créés depuis 1987 , l'attrait de la centralité est évident. Par ailleurs, sur les treize campings privés reccnsćs dans le département, neuf sont localisés dans cet espace.

L'offre en locatif a peu évolué et reflète mal les mutations actuelles de l'économie touristique du département. La répartition des gîtes relève davantage de la localisation de bâtis disponibles que d'une réelle demande. On notera justement qu'en 1996, certains prestataires ayant bénéficié récemment d'une subvention du Conseil général ont dû abandonner leur activité Gîtes de France avant la fin de leur engagement de dix ans, faute de résultat.

Les chambres d'hôtes, largement encouragées à l'ouverture du parc, sombrent désormais dans l'oubli et le Conseil général a même supprimé les subventions accordées pour la création de ces structures en 1997. Par ailleurs, depuis 1994, leur fréquentation chute régulièrement, même pour les chamabres d'hôtes commercialisées aux abords du Futuroscope où il paraît évident que l'offre hôtelière existante est suffisante pour satisfaire les clientèles de passage.

Au cours des dix dernières années, la création de cette centralité touristique a donc permis de rénover l'offre d'accueil du département et de lui donner un nouvel élan. Cette réorganisation, opérée au profit du parc du Futuroscope et des axes routiers proches, concerne dix communes qui détiennent aujourd'hui plus de 10500 lits marchand, soit $45 \%$ de l'offre du département. En outre les quatre cinquièmes de ces hébergements sont regroupés sur trois communes : la commune d'accueil du Futuroscope, Chasseneuil (4200 lits), la commune limitrophe de Jaunay-Clan (1620 lits) et la ville de Poitiers (2300 lits).

Dorénavant les dix communes qui composent ce pôle regroupent 701 hôtels pour 7500 chambres, soit $73 \%$ de l'offre hôtelière de la Vienne contre à peine $66 \%$ en 1992. Et les établissements du secteur du Futuroscope ont en moyenne plus de 60 chambres contre 22 dans le reste du département. A mesure de l'éloignement du Futuroscope, les campagnes faisant figure d'arrière-pays connaissent une réduction régulière de la diffusion touristique et leur parc d'hébergement est des plus réduits.

Évidemment, les nuitées sont avant tout concentrées sur le pôle touristique où les taux d'occupation sont les meilleurs de la région. Le volume des nuitées hôtelières du département atteignit 2 millions en 1996, soit un doublement depuis 1991, et bien sûr $85 \%$ de ces nuitées furent enregistrées dans la zone du Futuroscope. Ouvert en 1990, l'hôtel du Futuroscope, où sont commercialisées 290 chambres (pour une à quatre personnes), illustre remarquablement cette situation. Dès $1990 \mathrm{il}$ totalisa 26646 nuitées, deux ans plus tard plus de 112500 nuitées et en 1995, la fréquentation fut de l'ordre de 218000 nuitées. Le taux d'occupation annuel, qui est certainement l'un des meilleurs de la région, est de l'ordre de $80 \%$.

Par contre les nuitées en camping connaissent une progression plus modeste dans la zone du Futuroscope. Leur volume, de l'ordre de 360000 nuitées en 1996, cor- 
respond à $42 \%$ du total départemental. Cela s'explique notamment par la faiblesse de la durée des séjours qui n'est que de 2,5 nuitées par campeur.

L'impact du parc du Futuroscope, avec la consolidation de cette centralité, est incontestable sur l'organisation touristique de la Vienne. Les responsables du département veulent donc mettre à profit le succès du Futuroscope pour engager et aménager l'ensemble des campagnes de la Vienne; des territoires perçus en terme d'arrière-pays parfois d'angle-mort.

\section{III. - DU FUTUROSCOPE AUX CAMPAGNES AVOISINANTES : L'AMÉNAGEMENT TOURISTIQUE DE LA VIENNE}

Un rapide aperçu des différentes fréquentations enregistrées dans le département permet de saisir l'importance du décalage qui existe entre la centralité et le reste des territoires ruraux.

Le Futuroscope écrase l'activité touristique avec 2,8 millions de visiteurs. Ce pôle est consolidé par le tourisme urbain de la préfecture poitevine toute proche. La ville de Poitiers reçoit quelques centaines de milliers de visiteurs chaque année, parmi les édifices les plus fréquentés le Baptistère Saint-Jean (30 000 visiteurs), le musée Sainte-Croix (17000 visiteurs) et l'incontournable cathédrale Notre-Dame avec ses spectacles nocturnes. L'intérêt culturel est étoffé par le patrimoine touristique des quelques communes proches telles les abbayes de Ligugé (4500 visiteurs) et de Nouaillé (3500 visiteurs).

Enfin, au nord du Futuroscope, à une quinzaine de kilomètres, la base de loisirs de Saint-Cyr qui regroupe un plan d'eau et un parcours de golf accueille plus de 100000 vacanciers et excursionnistes.

Que dire de la fréquentation sur le reste du département ? On y recense 19 châteaux ouverts au public pour un total de 40000 visiteurs, 6 abbayes pour 36000 visiteurs et 38 musées pour moins de 24000 entrées. Deux exceptions à ce tableau : à Chauvigny, "le Château des Aigles » ouvert en 1996 qui reçut 32000 excursionnistes ct sur la commune de la Trimouille, "l'lle aux serpents » avec 52000 entrées pour la seconde année d'activité. Ces deux réalisations sont des projets structurants qui doivent donner naissance à de nouveaux pôles d'ancrage à partir desquels les flux de visiteurs pourraient être diffusés sur l'ensemble des campagnes du département.

\section{A) DES PROJETS STRUCTURANTS POUR UN MAILLAGE DU DÉPAR- TEMENT}

Les politiques conduites par le Conseil général visent à entreprendre un maillage du département avec la création de pôles secondaires qui feront figure de relais du Futuroscope au niveau des pays touristiques. Une approche classique avec une conception pyramidale dont le sommet est le parc d'attraction : le site touristique majeur de la Vienne est le produit d’appel touristique de l'ensemble du département.

Le site de Chauvigny correspond à une cité médiévale proche de Poitiers où d'importants efforts de restauration sont entrepris. Le projet structurant repose ici sur la création d'une nouvelle animation avec un spectacle d'aigles dans cette petite ville qui accueille habituellement près de 20000 visiteurs chaque été. Le château des évêques fut rebaptisé pour l’occasion "château des aigles ». Ensuite, une bonne campagne de promotion, en particulier à partir du Futuroscope, permit de 


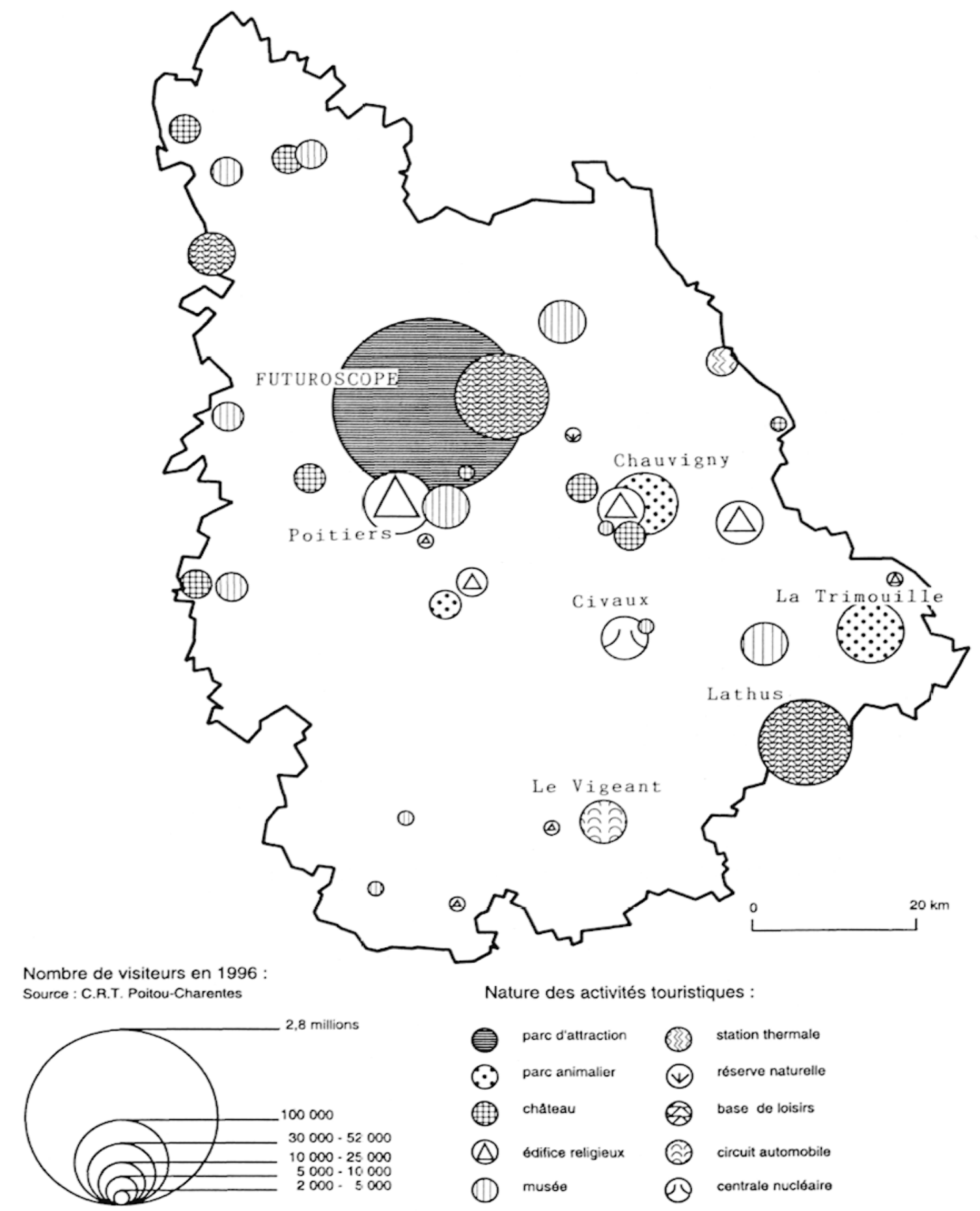

FIG. 3. - Les fréquentations touristiques dans la Vienne en 1996. 
draîner 12000 visiteurs supplémentaires en 1996. Ce projet reprend en fait des expériences conduites de longues dates à Rocamadour ( 150000 visiteurs par an) et à Cordes (dans le Tarn) et présente des animations identiques à celles du Puy-duFou, dans le département vendéen voisin.

Dans le cas de la Trimouille, petit chef-lieu relativement isolé du sud-est du département, le choix s'est porté sur une exposition de serpents, notamment en raison de la présence d'un pharmacien passionné de reptiles et possédant sa propre collection. Cette manifestation a permis à la commune de recevoir 45000 personnes dès 1995, mais les infrastructures d'accueil sont très réduites : un modeste camping, une petite auberge s'apparentant à un restaurant pour routiers et aucun hôtel.

En fait ces projets sont dits "structurants " car conçus pour draîner dès la première année d'activité 50000 visiteurs sur le site retenu. Mais ils ne s'accompagnent pas de nouvelle création d'hébergement sur le site d'accueil, ni d'équipement ludique qui pourront relever d'initiatives communales. Pour leur part les responsables départementaux du tourisme considèrent que l'offre de la centralité est suffisante, en particulier sur le plan de l'hôtellerie; ils ne tiennent donc pas à encourager la création de nouvelles structures d'accueil diffuses dans l'arrière-pays. Dans cette optique, les retombées locales ne peuvent donc être que superficielles. Il s'agit juste de créer des lieux d'animation qui, grâce à une bonne médiatisation. peuvent attirer des clientèles présentes au Futuroscope. Celles-ci accomplissent alors une excursion d'une demi-journée dans les campagnes pour rejoindre ensuite. la zone du Futuroscope ou quitter la Vienne.

Parmi les autres réalisations, il convient de présenter le circuit automobile de Formule 3 du Vigeant (homologué pour la Formule 1) qui doit devenir le pôle d'attraction sportif du département. Il reçoit actuellement près de 7000 visiteurs par an. Certes, l'investissement semble intéressant sur le plan économique, quoique coûteux en raison des frais constants qui doivent être consentis pour que le circuit reste homologué. La zone attenante au circuit regroupe désormais trois écuries automobiles et une société de stage de pilotage. En fait, les principaux atouts du site relèvent du coût d'un foncier bon marché et de l'espace disponible (aux abords du circuit, on recense tout juste une dizaine de personnes susceptibles d'être gênées par les nuisances sonores). Pour le moment, sur le plan touristique, le circuit n'a pas encore généré de développement spécifique.

D'autre projets sont en cours de réalisation comme «la vallée aux singes » à Romagne, dans le sud-ouest du département, dont l'ouverture est programmée pour le printemps 1998. Ce parc, vendu par un Néerlandais, où le visiteur découvre des singes en semi-liberté, est conçu sur le modèle de celui d'Apenheul (créé en 1971) qui reçoit annuellement 600000 visiteurs, mais ce dernier est situé à proximité d'Amsterdam.

Il y a incontestablement une certaine vogue pour ces parcs animaliers atypiques avec animations chez les collectivités locales, toujours persuadées de détenir le projet le plus original. On peut déjà recenser une douzaine de ces parcs dans le Sud-Ouest, présentés sous des appellations telles que «le donjon des aigles », «la falaise des aigles " ou encore " la forêt de singes " de Rocamadour.

Poursuivant rapidement l'énumération de ces différents projets dits structurants et en cours de réalisation, on peut citer celui de Civaux conçu autour de la centrale nucléaire dont le fonctionnement débute. Le projet porte ici sur l'ouverture d'une ferme aux crocodiles sur le modèle de celle de Pierrelatte (créée en 1992), mais on annonce aussi la création d'un vaste espace couvert qui réunira les plus vieux animaux du monde, parmi lesquels des tortues, des iguanes et des varans de Komodo. 
A Montmorillon, il est envisagé la création d'une "cité du livre et de l'écrit » qui aura pour vocation de devenir le pôle culturel du département, présentant l'écrit à travers les âges, des peintures ruspestres à internet en relation avec les infrastructures du Futuroscope. Le dernier projet adopté en 1997 concerne la commune de Lussac-les-Châteaux, destinée à devenir un centre historique consacré à la préhistoire avec l'ouverture au public de plusieurs grottes.

En fait des contenus de ces différents projets sont parfois assez inattendus, y compris pour les acteurs touristiques locaux qui découvrent dans la presse régionale des decisions du Conseil général. Les acquisitions foncières qui nécessitent ces différents projets sont déjà entreprises par les municipalités concernées. Certains y verront une inflation à l'approche d'échéances électorales avec des projets vendus "clé en main " qui n'ont besoin que de site d'accueil pour être rapidement fonctionnel.

\section{B) LES LIMITES DE CES AMÉNAGEMENTS}

Cette conception du développement touristique d'un département rural n'est pas sans comporter certaines limites.

- Pour l'heure ces aménagements planifiés et hiérarchisés semblent aller à l'encontre du développement local. Les projets dits structurants relèvent d'une politique du Département, lequel retient les projets, met en place les moyens nécessaires et choisit avec quelques conseillers généraux les sites susceptibles de recevoir ces réalisations sans consulter ni informer les acteurs locaux. D'aucuns soulignent d'ailleurs le peu d'impartiabilité dans le choix de ces sites ainsi que le manque d'intérêt accordé par les responsables départementaux aux initiatives proposées localement.

Pourtant certaines initiatives, comme celle conduite à Lathus, montrant l'intérêt de ces développements endogènes. Ainsi sur la commune de Lathus, située dans la vallée de la Gartempe, des initiatives associatives ont permis l'émergence d'un pôle touristique à partir d'une base de kayak. Une quarantaine de personnes travaillent actuellement dans ce centre proposant des prestations variées qui s'inscrivent dans leur environnement local ; aux activités sportives diversifiées s'ajoutent des formules destinées à sensibiliser les visiteurs aux paysages et activités agricoles qu'ils parcourent.

Le site de Lathus, doté de 400 lits (dont trois quarts en camping) attire plus de 100000 visiteurs annuellement. A l'image de cette réalisation qui s'inscrit dans la durée et qui génère d'intéressantes retombées pour l'économie locale, d'autres projets non élaborés dans le cadre de cette politique des pôles structurants mériteraient certainement un plus large soutien.

Dans un tel contexte, les projets décidés par le Département de connaissent pas une grande ferveur localement. Cette politique ne cherche pas à mettre en valeur les atouts de ces campagnes, il y a même une négation de leur identité rurale et paysanne. La nature des projets et les méthodes de développement adoptées relèvent davantage de la gestion d'espaces «libres ", d'arrière-pays peu prisés qu'on désire intégrer à la nouvelle centralité dans le cadre d'un maillage hiérarchisé.

- En dernier lieu il convient de s'interroger sur les limites de la polarité du Futuroscope à partir duquel repose toute la promotion touristique du Département ainsi que l'aménagement des campagnes.

'lout d'abord il semble que la fréquentation se stabilise autour de 3 millions de visiteurs. L'une des premières réactions face à cette stabilisation de l'activité est de 


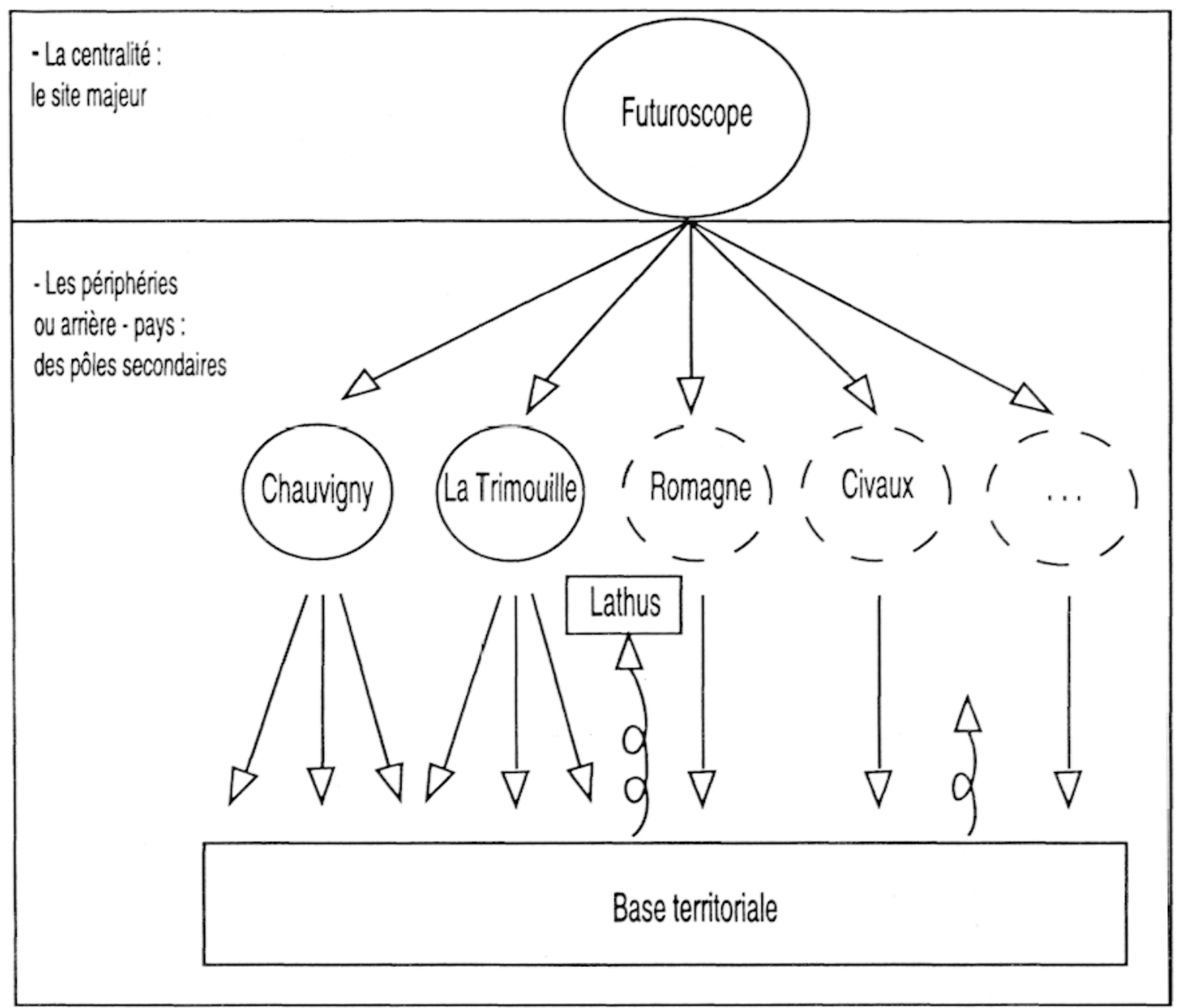

FIG. 4. - L'aménagement touristique du département de la Vienne.

favoriser une concentration des retombées financières au profit du parc. Ainsi les restaurateurs poitevins qui ont largement profité des visiteurs du Futuroscope, notamment en période estivale, observent depuis 1995 une diminution de ces clienthèles. En fait l'offre localisée aux abords du parc et surtout à l'intérieur de celui-ci $s$ 'est considérablement développée. Ainsi, en 1996, 60\% de visiteurs ont déjeuné dans le parc. La même année les restaurants du parc ont vendu 2 millions de repas assis, 1,8 millions de sandwichs et 1,1 millions de litres de boisson. Cette tendance va encore être confortée avec l'ouverture d'une pizzeria dans le parc en 1998.

Le parc d'attraction doit constamment renouveler sa clientèle qui est composé à $43 \%$ de groupes, parmi lesquels une bonne proportion de scolaires. Des espoirs reposent sur la construction de la nouvelle gare T.G.V. aux abords du Futuroscope avec la réalisation d'une passerelle permettant d'accéder directement au parc.

Les clientèles doivent également être fidélisées. En ce sens une nouvelle grille tarifaire vient d'être adoptée avec la mise en place d'une formule «futuropass» dont l'achat donne un accès permanent au parc pendant trois ans.

Enfin, en dépit des performances des politiques commerciales du Futuroscope, se pose le problème fondamental de l'originalité du parc. L'originalité actuelle résulte de l'importance des techniques innovantes concentrées dans cet espace qui reste unique en Europe. Cependant on peut s'interroger sur la durabilité de cet amé- 
nagement sachant que s'annonce une concurrence irrémédiable avec l'apparition de mini-parcs qui va entraîner la diffusion rapide de ces techniques à une plus grande échelle. La multiplication des petites unités à thème présentant les technologies les plus récentes est déjà une réalité en Amérique du Nord et au Japon. L'exemple du procédé Imax 3D qui permet la projection de film en relief est révélateur à cet égard. Ce fut longtemps l'une des originalités du Futuroscope et de la Cité des Sciences et de l'Industrie, désormais le quartier de la Défense est également équipé de ce procédé Imax et certains grands centres commerciaux en ont commandé l'installation (2). Toujours à Paris, cette tendance est aussi perceptible avec l'ouverture du centre Séga couplé au restaurant Mac Donald's (place d'Italie). La situation est identique à Londres avec l'immeuble Séga de Piccadilly Circus.

Dans un tel contexte, être toujours à la pointe de l'innovation sera de plus en plus lourd à gérer pour des grosses infrastructures comme le parc du Futuroscope qui auront quelques difficultés pour continuer à affirmer leur originalité face à l'ouverture de ces petites unités plus faciles à rentabiliser et implantées au cœur des plus gros foyers de consommateurs.

\section{CONCLUSION}

Les efforts engagés depuis la fin des années 80 ont permis la création d'une véritable centralité touristique qui faisait défaut au département de la Vienne. Elle repose sur le pôle événementiel du Futuroscope à partir duquel est conçu l'aménagement du reste du département.

La Vienne connaît désormais des fréquentations estivales d'une ampleur notable. Toutefois, l'activité reste largement dominée par un tourisme de passage, les pôles structurants destinés à diffuser les visiteurs dans les campagnes favorisent tout au plus la réalisation de courts séjours.

Pour le moment les campagnes susceptibles d'accueillir des vacanciers séjournant plus longtemps sont insuffisamment valorisées à l'image des vallées de la Vienne et de la Gartempe. Dans le cadre de l'aménagement du département, elles font figure d'arrière-pays voire d'angle-mort et sont perçues en terme d'espacesupport disponible et peu coûteux.

Enfin, à l'échelle régionale, le dynamisme du parc du Futuroscope permet d'envisager une nouvelle articulation entre la centralité poitevine et la côte charentaise : des touristes marquent une étape à Poitiers avant de s'installer sur le littoral, d'autres réalisent une excursion vers l'intérieur, jusqu'au Futuroscope, durant leurs vacances balnéaires. L'amplification de ces flux de passage favorise l'émergence de nouveaux centres d'intérêt touristique, notamment dans les campagnes deuxsévriennes, qui se greffent sur l'axe Futuroscope-le littoral atlantique.

(2) Cf. Davis (S.G.), 1998. - «L'espace urbain perverti par les loisirs " in Le Monde diplomatique, janvier 1998. 